\title{
Assessment of Continuous Vaginal Logger-Based Temperature Monitoring in Fever-Induced Gilts
}

\author{
${ }^{1}$ Mariana Schmidt, ${ }^{1}$ Christian Ammon, \\ ${ }^{2}$ Peter Schon, ${ }^{2}$ Christian Manteuffel and ${ }^{1}$ Gundula Hoffmann \\ ${ }^{1}$ Department of Engineering for Livestock Management, \\ Leibniz-Institute for Agricultural Engineering Potsdam-Bornim (ATB), Potsdam, Germany \\ ${ }^{2}$ Department of Behavioral Physiology, \\ Leibniz-Institute for Farm Animal Biology Dummerstorf (FBN), Dummerstorf, Germany
}

Received 2013-04-23, Revised 2013-05-22; Accepted 2013-05-30

\begin{abstract}
The aim of this study was to identify a low risk method to induce fever in gilts and to evaluate vaginal sensors for temperature monitoring. Therefore, a rhinitis atrophicans vaccine was used to induce fever to evaluate sensors and behavioral patterns. During 11 trials, two of four animals were injected with $2 \mathrm{~mL}$ of the vaccine Porcilis AR-T DF (Intervet) and the other two animals were treated with $2 \mathrm{~mL}$ of $0.9 \% \mathrm{NaCl}$ as controls. A temperature logger (TRIX-8, LogTag Recorders, Auckland, New Zealand) was used to continuously monitor the vaginal temperature. Additionally, rectal temperatures were measured four times daily. The water consumption, food intake and locomotion behaviors of the animals were analysed one day before treatment and during the day of the treatment. The vaccine induced fever in all gilts, which occurred approximately $5.87 \mathrm{~h}$ after vaccination. The vaginal logger temperatures and the rectal temperatures showed a linear correlation in 21 vaccinated gilts $(\mathrm{r}=0.86 ; \mathrm{p}<0.0001)$ and 22 non-vaccinated gilts $(\mathrm{r}=0.65$; $\mathrm{p}<0.0001)$. The vaginal logger can be continuously used to measure the core temperature. Water intake decreased $(p<0.0001)$ in the gilts with fever, whereas food intake did not change $(p=0.9411)$. The vaccinated gilts spent $79 \pm 16$ more minutes per day lying after the vaccination $(p<0.001)$ in comparison to the day before the vaccination. Treatment with this vaccine provides the opportunity to induce fever over a short time period $(5.13 \mathrm{~h})$ and mild sickness behavior in gilts to investigate disease indicators.
\end{abstract}

Keywords: Gilts, Vaccination, Temperature, Behavior, Disease Detection, Fever

\section{INTRODUCTION}

Pathogens, adjuvants and vaccines are typically employed to induce fever in research studies or for antibody production (Harden et al., 2011; Leenaars and Hendriksen, 2005; Schaefer et al., 2004; Stills, 2005). Fever is triggered by the release of endogenous pyrogens from different regions of macrophage-like cells. These pyrogens include cytokines IL-1 and IL-6, which act at the level of the anterior hypothalamus to raise the thermoregulatory set point (Kluger et al., 1998). However, fever is also one of the main responses to Complete Freund's Adjuvants (CFA) (Melchior et al., 2004; 2005) or to Lipopolysaccharide (LPS) (De Groot et al., 2007; Wright et al., 2000) in piglets. Often the injection routes are not clear and painful processes occur after the injection (Leenaars and Hendriksen, 2005; Stills, 2005). Nagai et al. (1957), who injected CFA into guinea pig nuchal musculature, demonstrated that fibrotic changes were found in the lung. A study by Banji et al. (2011) demonstrated that arthritis was induced in rats by a CFA injection. Furthermore, rabbits were injected with CFA in the marginal ear vein, with significant damage to the lung as a result (Brooks et al., 1978). LPS activates the Toll-Like Receptor 4 (TLR-4), which results in the production of the pro-inflammatory cytokine IL-1, an

Corresponding Author: Mariana Schmidt, Department of Engineering for Livestock Management,

Leibniz-Institute for Agricultural Engineering Potsdam-Bornim (ATB), Potsdam, Germany 
important cytokine for the induction of sickness behavior (Konsman et al., 2002). LPS can also have negative effects, such as endotoxemia (Noh et al., 2012) and can cause periodontal inflammation in rats (Dumitrescu et al., 2004), synovitis in horses (Loon et al., 2010) and abortion in gilts (Cort and Kindahl, 1990). However, the common physiological and pathological changes that occur due to LPS treatment are transient fever, depressed feed intake, changes in the concentrations of acute phase proteins and activation of the hypothalamic-pituitary-adrenal axis (Kelley et al., 1994). Furthermore, animals that have been treated with LPS have to be killed in Germany (EC, 2002) because these animals are not allowed to be used for food production.

In the present study, a vaccination was used to induce fever while circumventing these undesirable side effects. A comparison of the package leaflets of 9 vaccines for pigs, regarding undesirable side effects such as fever induction, indicated that the Rhinitis Atrophicans (RA) vaccine can lead to a temperature increase of up to $2.0^{\circ} \mathrm{C}$.

Methods to continuously measure the body core temperature of cattle currently exist. For example, Timsit et al. (2011) used a reticulo-rumen bolus to measure the core temperature in cows and Vickers et al. (2010) and Hoffmann et al. (2013) used vaginal loggers for this purpose. Because a rumen bolus is not appropriate for pigs because of anatomical reasons, a climate monitoring device was used and validated as a vaginal logger in our study.

Infected animals exhibit a distinct behavioral pattern. Therefore, it is also important to record their standing and lying behavior and water and food intake for disease and pain detection (Fraser and Quine, 1989; Hart, 1988; Weary et al., 2006). Animals suffering from an illness are often anorectic, lethargic and uninterested in social interactions (Johnson, 2002). For example, water and food intake are reduced in rats injected with endotoxin (Harden et al., 2011) and all sick animals typically increase the amount of time that they rest (Hart, 1988). These traits can be a sign of illnesses and can be useful for monitoring the general health condition of an animal.

The aim of the present study was to identify a method that increases the core body temperature to at least $39.5^{\circ} \mathrm{C}$ without the aforementioned side effects and to continuously monitor the animal's behavior to investigate how fever changes behavioral patterns. By performing continuous monitoring, direct and indirect correlations between body temperature and behavior can be studied in greater detail. In particular, we expect a clear pattern change at a rectal temperature above $39.5^{\circ} \mathrm{C}$, defined as fever by Blood and Henderson (1983).

\section{MATERIALS AND METHODS}

\subsection{Animals}

The study was conducted at the experimental pig unit of the Leibniz-Institute for Farm Animal Biology (FBN, Dummerstorf, Germany) with 55 German Landrace gilts in 14 trial runs from May to December 2012. The gilts were from 208-to 215-days-old. Four gilts were tested in each trial run. The animals were housed on a solid concrete floor in individual pens side by side, leaving one pen in between empty for the installation of electronic measurement devices. Each pen measured $0.6 \times 2.2 \mathrm{~m}$. The feed consisted of gilt rearing feed pellets (Trede and von Peine, agricultural trade and feed mills, Dammfleth, Germany). The animals were fed twice a day with $1300 \mathrm{~g}$ of pellets and water was available ad libitum. During the trial, the animals were examined for signs of vaginal discharge caused by the logger. Afterwards, the gilts were culled or kept for breeding.

The RA vaccine can lead to an increase in temperature of approximately $2^{\circ} \mathrm{C}$, furthermore the gilts have never been vaccinated with this vaccine before. Therefore we decided to choose the RA vaccine for the fever stimulation.

\subsection{Treatment \\ 2.2.1. Dose Test}

To determine the dose for the main trial, a dose test was first conducted. For this dose test, three groups of four gilts were treated with three different doses of a commercial vaccine, respectively and one animal out of each group was treated with a placebo. Altogether 12 gilts were part of the dose test. The commercial vaccine Porcilis AR-T (Intervet International B.V., Boxmeer, The Netherlands; RA vaccine) was administered one time to randomly chosen single gilts using $2 \mathrm{~mL}$ (single dose), $4 \mathrm{~mL}$ (double dose) and $6 \mathrm{~mL}$ (triple dose). A dose of $6 \mathrm{~mL}$ of $0.9 \%$ sterile $\mathrm{NaCl}$ solution (Selectavet Dr. Fischer GmbH, Weyarn-Holzolling, Germany) was administered to the fourth gilt.

\subsection{Main Trial}

The main trial was conducted with 43 animals. During trial run 14, one sow had to be excluded from the trial. During the main trial, the same vaccine was administered one time to two single gilts using a $2 \mathrm{~mL}$ dose. A dose of $2 \mathrm{~mL}$ of $0.9 \%$ sterile $\mathrm{NaCl}$ solution was administered to the two other gilts (control). The treatment was conducted on the third day of each trial run between 09:00 and 09:30 in the morning by an intramuscular injection of the corresponding dose into the right side of the neck and directly behind the ear. 


\subsection{Measurements}

The Rectal Temperature (RT), Vaginal Temperature (VT), lying behavior and water and feed intake were measured for four days. On day three, the gilts were injected with the vaccine or $\mathrm{NaCl}$. The data for the day of treatment (day three) were compared to the day before (day two).

During all trial days, the RT was measured four times daily (09:00 a.m., 11:00 a.m., 02:00 p.m., 04:00 p.m.) using a digital thermometer (Microlife MT 1831, Lifeware Rheintal AG, Widnau, Switzerland).

To avoid measurement errors due to too low insertion depths or dirt, the measurement was performed twice with a few minutes delay. Only the higher of both values was included in the evaluation. The VT was measured continuously from day two to day four using a microclimate monitoring device designed for industrial purposes with an external temperature probe (TRIX-8 recorder with ST100S-15 probe, LogTag Recorders, Auckland, New Zealand). The temperature probe was inserted into the vagina with an insertion depth between 5 to $10 \mathrm{~cm}$. The logging device and sensor cable were fixated at the back and the backside of the gilts using adhesive tape (Fig. 1). The Vaginal Logger (VL) took temperature data every three minutes. In the event of the probe being pushed out of the vulva, e.g., by excretion, it was cleaned and reinserted.

To record the lying behavior, a Video Graphics Array (VGA) resolution webcam (IC 3005, Edimax, New Taipei City, Taiwan) was installed in each pen. An additional webcam of the same type monitored all animals at the same time from an elevated position. The video stream was recorded as mpeg-4 video for ten hours per day from 07:00 a.m. to 12:00 and from 12:01 until 05:00 p.m. throughout the entire trial run. Video analysis was evaluated by visual inspection using Windows Media Player (Microsoft Corporation, Redmond, USA) and included the recording of time and duration of the animals' postures based on a network time protocol synchronised timestamp overlay within the video. Postures were discriminated between lying, sitting and standing. The time that the gilts spent in various positions during the day of the vaccination was compared to the day before.

For the measurement of water consumption, each pen was equipped with a bite ball valve (AquaGlobe $\mathrm{AB}$, Uppsala, Sweden) and a digital flow meter (FCH-m-POM, B.I.O-TECH e.K., Vilshofen, Germany). The overall water pressure could be regulated by a $1 / 2$ " ball valve.

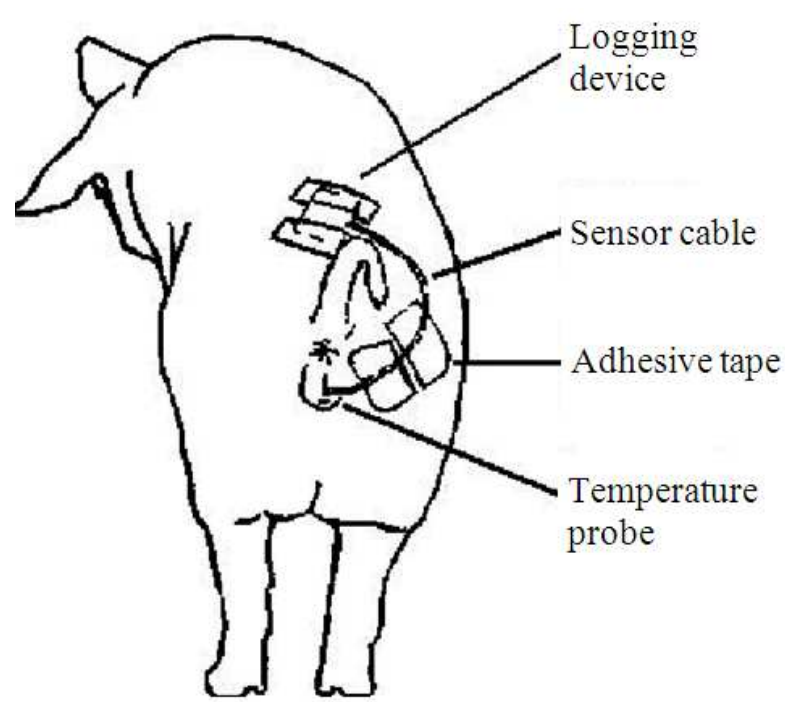

Fig. 1. Placement of the vaginal logger at the rear of a sow (probe is intravaginal)

The water consumption was recorded continuously every day in $60 \mathrm{~mL}$ steps using NI 6008 USB data acquisition devices in combination with software developed by ourselves using NI Labview (both National Instruments, Austin, USA). For both groups, the water consumption was compared between the day before vaccination and the day of the treatment.

Feed intake was measured by weighing the feed remains in removable troughs after a feeding time of twenty minutes. The accuracy of the scale was $\pm 50 \mathrm{~g}$. The feed rate was measured starting with trial run eight using a stopwatch (Compact II, servoprax $\mathrm{GmbH}$, Wesel, Germany). The gilts were fed twice a day (08:00 am and 01:00 p.m.) during the dose test. During the main trials, the afternoon feeding time was shifted (01:00 p.m., 03:30 p.m., 03:30 p.m., 01: 00 p.m.) to maximise the expected effect of the vaccination at 03:30 in the afternoon of day three.

\subsection{Statistical Analyses}

The statistical analyses were performed using SAS 9.3 (SAS Institute Inc., Cary, NC, USA).

Temperatures of the VL below $35^{\circ} \mathrm{C}$ were defined as no body core temperature by the authors because the logger slipped out the vulva. Therefore, we excluded those measurements for the statistical analyses.

\subsection{Dose Test}

A mixed linear model was used to test the differences between doses regarding VL temperature increase. Observations were mean temperature per hour and sow, 
fixed effects beside the intercept $\mu$ were dose (D; $\mathrm{i}=1, \ldots, 4), \mathrm{h}(\mathrm{H} ; \mathrm{j}=1, \ldots, 24)$ and interaction between dose and hour. Random effects included sow effect (S; $\mathrm{k}=1, \ldots, 11)$ and interaction between sow, hour and the independent normally distributed residual $\varepsilon$. This resulted in the model shown in Equation (1):

$$
y_{i j k}=\mu+D_{i}+H_{j}+(D \times H)_{i j}+S_{k}+(S \times H)_{j k}+\varepsilon_{i j k}
$$

\subsection{Main Trial}

Pearson correlation coefficients between rectal and vaginal temperatures were calculated separately for both treatments ( $\mathrm{RA}$ vaccine and $\mathrm{NaCl}$ ) in the main trial of the study. After the treatment, the time until the temperature exceeded $39.5^{\circ} \mathrm{C}$ was measured with the vaginal logger and averaged to obtain the average time it took to induce fever. Starting from this point in time, the time span was measured until the temperature fell below $39.5^{\circ} \mathrm{C}$ again. This time span was also averaged across all gilts to obtain the average fever duration.

Furthermore, a Bland-Altman plot was performed to evaluate the vaginal logger data graphically, according to the method of Bland and Altman (1999) and recommended by Grouven et al. (2007), for comparisons of both of the methods under investigation. This statistical procedure is especially recommended for comparing two methods because it not only considers the average difference (bias) but also puts emphasis on the variation of the differences (diff) between pairs of measurement values. The differences between the measurements with both methods are plotted against the arithmetic mean of the two methods. The bias is provided as the mean difference and the $95 \%$ limits of agreement are provided by diff $\pm 2 \mathrm{~s}$, where " $\mathrm{s}$ " is the standard deviation of the differences.

The respective durations of standing and lying were tested for differences between the treatments using mixed linear models. For each trait, the time per day in seconds was summed. The duration of sitting was tested using a generalized linear mixed model assuming a Poisson distribution and using a Log link function. Fixed effects were the general mean $\mu$, trial $(\mathrm{TR} ; 1=1, \ldots 11)$, dose $(D ; i=1,2)$, point in time (PIT; $m=1,2$ referring to measurements before and after vaccination, respectively) and interaction between D and PIT. The random sow effect $(\mathrm{S} ; \mathrm{k}=1, \ldots, 43)$ was also included. Residuals were assumed to be independent and $\mathrm{N}\left(0, \sigma^{2}\right)$ distributed for standing and lying, resulting in the model in Equation (2):

$$
y_{i j m l}=\mu+T R_{l}+D_{i}+P I T_{m}+(D \times P I T)_{i m}+S_{k}+\varepsilon_{i j m l}
$$

The influence of the treatments on sitting duration was tested using a generalized mixed linear model. In addition to the intercept $\mu$ fixed effects of TR, D, PIT and the interaction between D and PIT, the random sow effect $\mathrm{S}$ was also included in the model. For sitting duration, a Poisson distribution was assumed. A Log link function was used to link the distribution parameter to the linear predictor $\eta$ given in Equation (3):

$$
\eta_{i j m l}=\mu+T R_{l}+D_{i}+P I T_{m}+(D \times P I T)_{i m}+S_{k}+\varepsilon_{i j m l}
$$

For testing treatment effects on water consumption per day in $\mathrm{mL}$, the same model was assumed. Hence, the linear predictor $\eta$ from equation (3) was used.

Differences in feed intake were tested using a generalized linear mixed model. The observed feed remains divided by total feed amount resulted in the percentage of remaining feed and, hence, were assumed to follow a binomial distribution. A logit function was used to link the distribution parameters to the linear predictor. The linear predictor $\eta$ included the general mean $\mu$ and the fixed effects of TR, D, temperature condition (TC; $n=1,2$ referring to temperatures up to and above $39.5^{\circ} \mathrm{C}$, respectively), the interaction between dose and temperature condition, random sow effect and logistically distributed residual $\varepsilon$, as shown in Equation (4):

$\eta_{i k \ln }=\mu+T R_{l}+D_{i}+T C_{n}+(D \times T C)_{i n}+S_{k}+\varepsilon_{i k \ln }$

Differences in the duration of feed intake were tested using the same model, except that the interaction between D and TC was excluded and only 9 trials were considered. The observed variable was the amount of time for feeding in each sow divided by a maximum time of $20 \mathrm{~min}$. For all of the tests using the models (equations 1 to 4), hypotheses were tested at a significance level of 0.05 . P-values from pairwise tests between effect levels were adjusted for multiple testing using the SIMULATE option to comply with the global significance level.

\section{RESULTS}

\subsection{Dose Test}

Average temperatures per hour \pm Standard Error (SE) were $\quad 38.46 \pm 0.09, \quad 39.54 \pm 0.09, \quad 39.07 \pm 0.11 \quad$ and $38.89 \pm 0.13^{\circ} \mathrm{C}$ for the $\mathrm{NaCl}$ control (referred to as 0 in Table 1), $2 \mathrm{~mL}$ dose, $4 \mathrm{~mL}$ dose and $6 \mathrm{~mL}$ dose, respectively. 


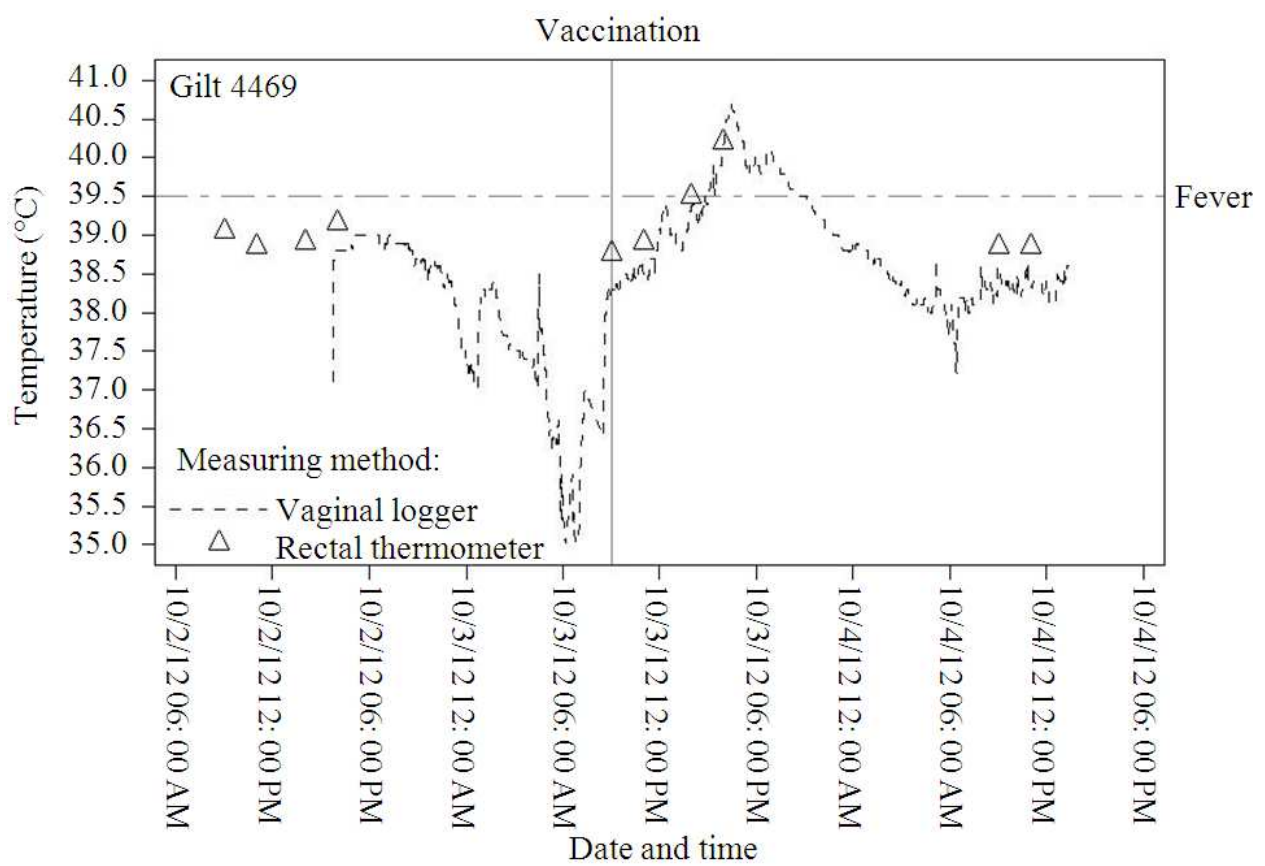

Fig. 2. Course of rectal and vaginal temperatures during the three days of the trial for one sow with the vaccination and the fever limit marked

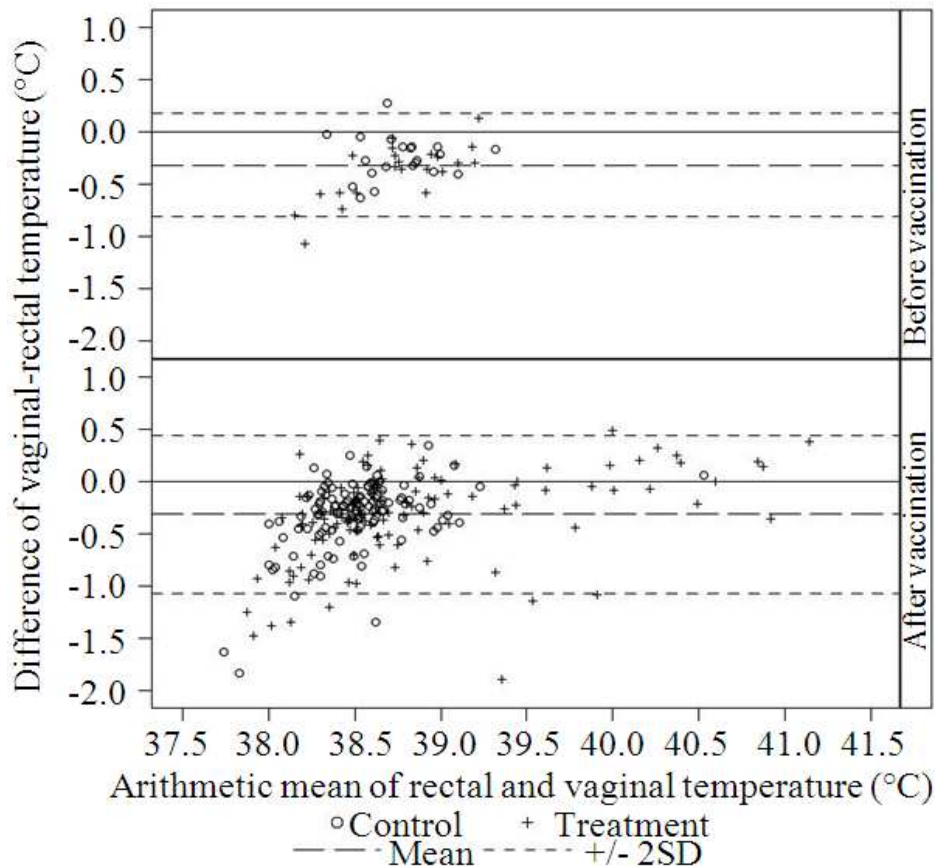

Fig. 3. Bland-Altman plot of the values for the vaginal temperatures compared to the rectal temperatures, based on the observations in the main trial (43 gilts) 


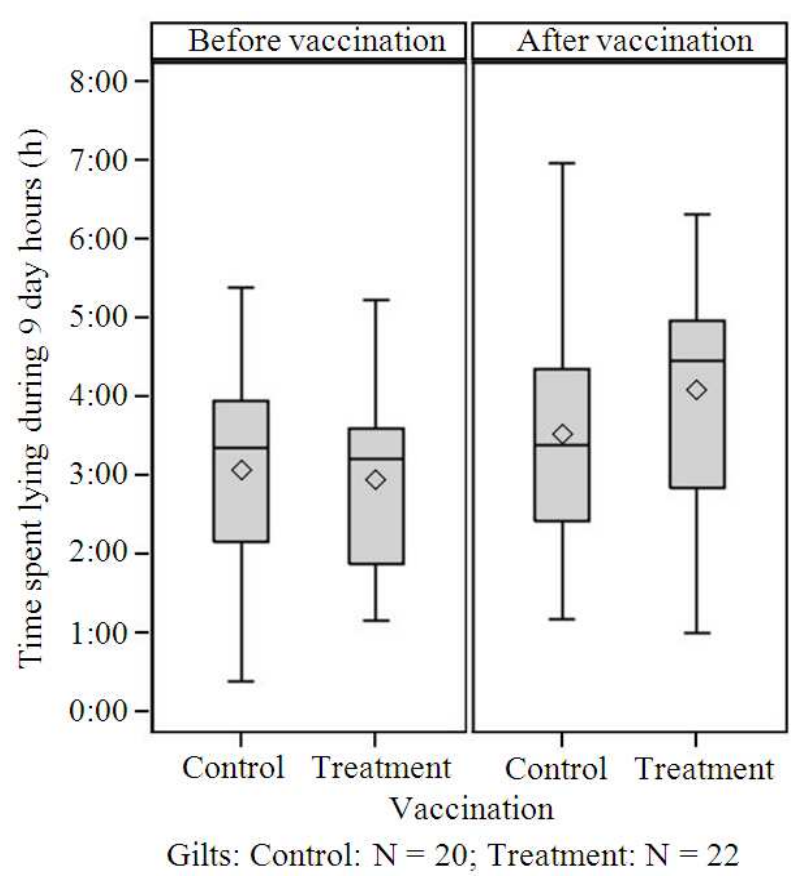

Fig. 4. Lying time of the gilts (42 gilts) before and after treatment with $\mathrm{NaCl}(0)$ or with the vaccine (2)

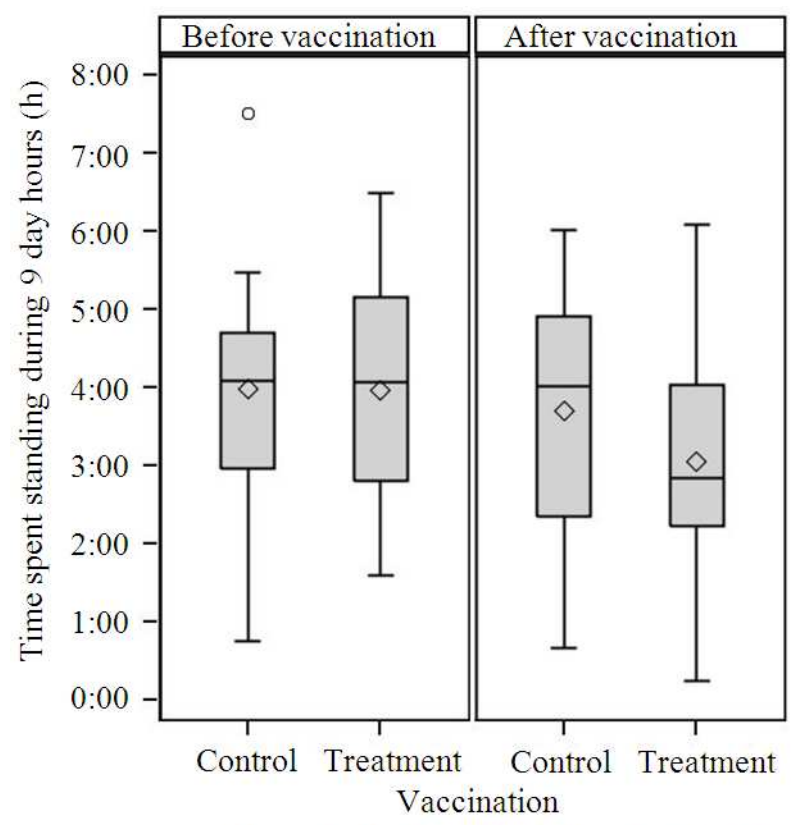

Gilts: Control: $\mathrm{N}=20$; Treatment: $\mathrm{N}=22$

Fig. 5. Time spent standing (42 gilts) before and after treatment with $\mathrm{NaCl}(0)$ and with the vaccine (2)
Table 1. The least squares means, estimated differences, standard error, degrees of freedom and adjusted Pvalue differences for the temperatures per treatment in the dose test

\begin{tabular}{|c|c|c|c|c|}
\hline $\begin{array}{l}\text { Difference } \\
\text { P-value }\end{array}$ & Estimate & $\begin{array}{l}\text { Standard } \\
\text { error }\end{array}$ & $\begin{array}{l}\text { Degreeso } \\
\text { ffreedom }\end{array}$ & Adjusted \\
\hline Dose 0 -Dose 2 & -1.09640 & 0.1546 & 219 & $<0.0001$ \\
\hline Dose 0 -Dose 4 & -0.67730 & 0.1768 & 219 & 0.0010 \\
\hline Dose 0 -Dose 6 & -0.65590 & 0.1886 & 219 & 0.0033 \\
\hline Dose 2-Dose 4 & 0.41910 & 0.1773 & 219 & 0.0828 \\
\hline Dose 2-Dose 6 & 0.44050 & 0.1891 & 219 & 0.0894 \\
\hline Dose 4-Dose 6 & 0.02137 & 0.2077 & 219 & 0.9999 \\
\hline
\end{tabular}

As can be observed in Table 1, all treatments lead to a significant increase in body temperature compared to the control. Between the doses, no significant differences occurred. Hence, the lowest dose was chosen for the following trials.

\subsection{Main Trial}

The linear correlation between the RT and VT was $r=0.65(p<0.0001)$ for the 22 non-vaccinated gilts $(\mathrm{n}=136)$ and $\mathrm{r}=0.86(\mathrm{p}<0.0001)$ for the 21 vaccinated gilts $(\mathrm{n}=152)$.

On average, an increase of the VT above $39.5^{\circ} \mathrm{C}$ could be detected after $5.87 \mathrm{~h}$ post-vaccination. An example of the temperature increase of one gilt is shown in Fig. 2. The temperature was again below $39.5^{\circ} \mathrm{C}$ after $11.20 \mathrm{~h}$ post-vaccination. Therefore, on average, the fever period lasted $5.13 \mathrm{~h}$.

A Bland-Altman analysis was conducted to validate the use of the vaginal loggers. The Bland-Altman plot (Fig. 3) of the difference between VT and RT indicates that most values were within 2 Standard Deviations (SD). The majority of the outliers can be found in the low temperature area, except for one.

The behavioral analysis showed differences between the time before and after vaccination (Fig. 4 and 5). The gilts treated with the vaccine spent $79 \pm 16 \mathrm{~min} /$ day more lying post-vaccination compared to the day before the treatment $(\mathrm{p}=0.0003)$ and $52 \pm 18 \mathrm{~min} /$ day less standing $(p=0.0251)$. The vaccinated gilts spent $3.29 \mathrm{~min}$. more sitting compared to the day before $(\mathrm{p}<0.0001)$, whereas the sows that were injected with $\mathrm{NaCl}$ spent $6.57 \mathrm{~min}$. more sitting per day $(\mathrm{p}=<0.0001)$. Water intake clearly demonstrated a rhythmic day by the gilts. The highest water intake for the gilts was between 12:00 PM and 4:00 PM and water intake changed significantly for both the vaccinated $(\mathrm{p}<0.0001)$ and non-vaccinated $(\mathrm{p}<0.0001)$ gilts. 


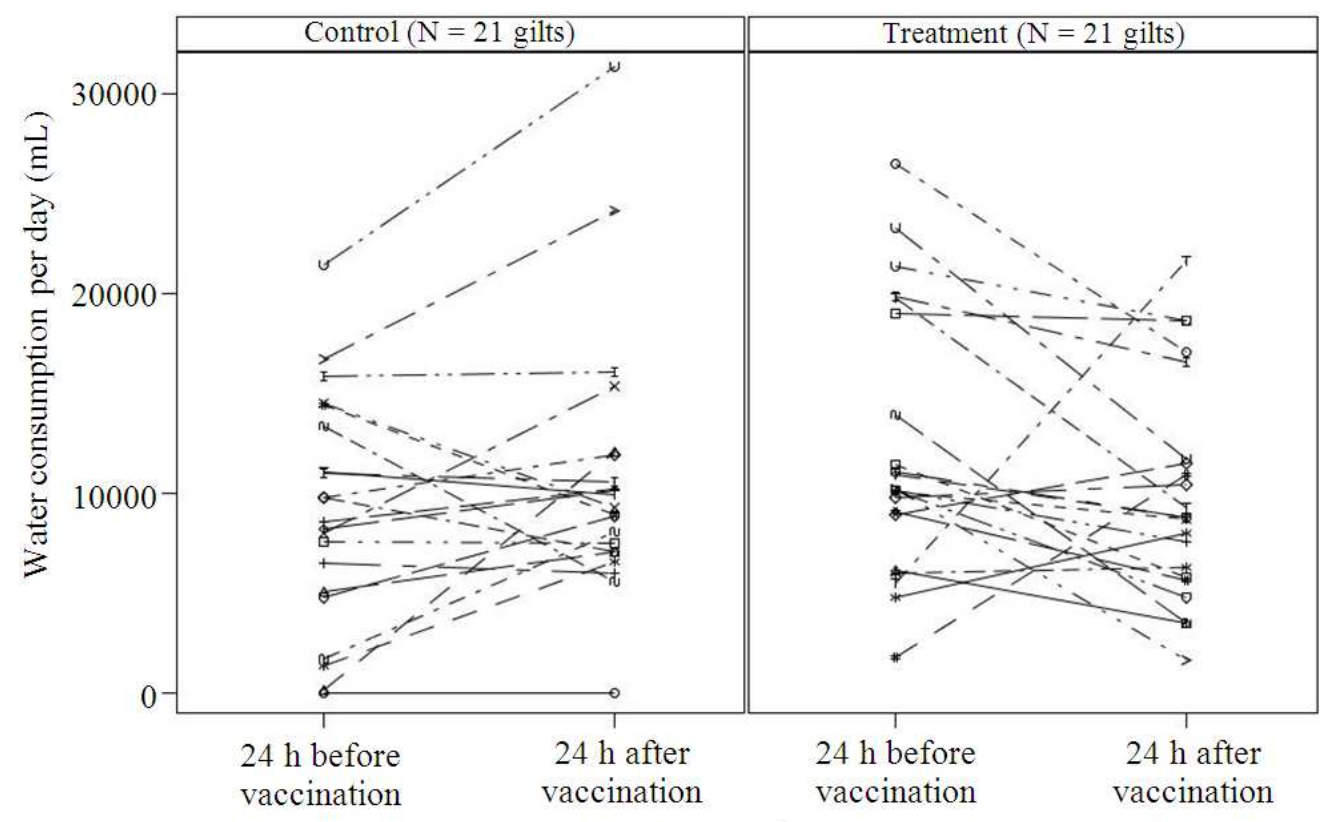

Measuring day

Fig. 6. Water consumption per day for all non-vaccinated and all vaccinated gilts. The first dot marks $24 \mathrm{~h}$ before vaccination and the second dot marks $24 \mathrm{~h}$ post-vaccination

The difference between both groups was that the nonvaccinated gilts drank $1.1 \mathrm{~L}$ more during a 24-h period compared to the day before, whereas the vaccinated gilts drank 2.01 L less compared to the day before (Fig. 6).

Two of the vaccinated gilts did not eat at all during the entire trial. The temperatures of these gilts were $41.2^{\circ} \mathrm{C}$ and $41.6^{\circ} \mathrm{C}$ at 4:00 PM. The feeding behavior of the other gilts displayed no changes. Food intake was not influenced by vaccination or fever ( $p>0.05)$. In addition, the duration of the feed intake was not influenced by vaccination or fever. There was only one significant difference between trial $12(18.67 \pm 0.67 \mathrm{~min})$ and all of the other trials (on average $11.4 \pm 3.38$ min (mean \pm SE) per feed intake).

No side effects on the animals' health due to the logger were observed during the trial (vaginal discharge).

\section{DISCUSSION}

The dose test demonstrated that there was a significant difference in the temperature increase between the gilts treated with $\mathrm{NaCl}$ and the gilts treated with the vaccine. Although there was an increase in temperature for the gilts treated with the $4 \mathrm{~mL}$ and $6 \mathrm{~mL}$ doses, it was not significant compared to the $2 \mathrm{~mL}$ dose.
A reason for this increase could be that a dose of $2 \mathrm{~mL}$ is sufficient to elicit an immune reaction and that a certain level of saturation is reached.

Temperatures measured with a rectal thermometer are often not accurate enough because of inadequate insertion depth (Naylor et al., 2012). A study by Burfeind et al. (2010), who recorded temperature data from cows, determined that rectal temperatures are higher when the probe is inserted deeper into the rectum. We tried to minimise measurement errors by performing the measurements twice with a certain time lag. Continuous measurements with a fixed VL can be used as a measure of body temperature in dairy cows (Suthar et al., 2013) and could be helpful for avoiding misleading data, as explained by Burfeind et al. (2010). In a study by Soede et al. (1997), a vaginal sensor was used in sows, indicating that it is possible to measure the body core temperature with such a logger. Similarly, our results demonstrate that the VT is highly correlated to the RT. One reason for outliers in the Bland-Altman plot in the negative area could be that, in some animals, the VL was not inserted deeply enough before it slid out of the vulva, especially when the animals urinated and defecated. Further investigations will be required to find a solution for fixation of the VL to improve the method, 
specifically to prevent it from slipping out. However, the VL caused no side effects on the animals' health and core temperatures could be measured. It remains to be determined if this method will be a useful option for research studies. However, this temperature measurement method is not an option for commercial pig farms because the risk of ascending infections would be too high.

It could be shown that the Porcilis AR-T DF vaccination led to an increase in the body temperature of the gilts. Although the increase lasted no longer than a few hours, some behavioral patterns were altered. Furthermore, this method provides the opportunity to induce fever without the common side effects of using methods such as CFA and LPS, which have been described by other authors (Brooks et al., 1978; Leenaars and Hendriksen, 2005; Stills, 2005).

The lying behavior increased in the vaccinated gilts. In conclusion, the standing behavior decreased in the vaccinated animals. In addition, the sitting behavior of the vaccinated animals increased. In particular, the nonvaccinated gilts spent more time sitting compared to the vaccinated gilts. An explanation for this finding could be that the vaccinated gilts spend more time lying on the whole than the non-vaccinated gilts. The vaccine contains pyrogenic substances and possibly leads to an IL-1 increase (Kluger et al., 1998). This is one of the major cytokines and acts on the hypothalamus. This includes, next to other symptoms, increased sleep (Tizard, 2008). In contrast, pigs generally respond to increasing ambient temperatures by modifying their lying, excretion and wallowing behaviors (Huynh et al., 2005). Both factors could be an explanation for our results.

The water intake of the gilts was altered during the present trial. The gilts that were treated with the vaccine drank less compared to the day before, whereas the gilts injected with $\mathrm{NaCl}$ drank more compared to the day before. This result is in agreement with the results of Harden et al. (2011), who described that rats injected with LPS showed anorexia. Additionally, laboratory rodents injected with proinflammatory cytokines and LPS showed a decrease in food and water intake (Dantzer, 2001). There was no obvious reason for the increase in water intake by the $\mathrm{NaCl}$-vaccinated gilts.

In the present study, the food intake of the gilts did not decrease $(p>0.05)$ after vaccination. Sick individuals usually decrease feeding (Weary et al., 2009) to promote recovery (Johnson, 2002). In their trial, McCarthy et al. (1985) found that food intake is suppressed during the infection of rats and is mediated by the release of IL-1. Furthermore, it has been shown that interleukin-1ß (IL-
13) has an influence of the motivation to eat. Rats infected with Mycoplasma were lethargic and anorexic and showed body mass stunting (Swanepoel et al., 2011). Waldron et al. (2006) demonstrated that the feed intake of lactating dairy cows declined in response to LPS. Illnesses and the immune response can also affect the water and food uptake of mammals (Dantzer, 2001; Weary et al., 2009). In spontaneously RA-affected pigs, toxin-producing strains of Pasteurella multocida are the decisive pathogen factor (Zimmermann and Plonait, 2004). The RA vaccination contains inactivated Bordetella multocida cells and a protein component of the $P$. multocida dermonecrotic toxin, which also influences IL secretion and, therefore, has an influence of the animal's sickness behavior. In response to vaccination, we found a modified water intake but a predominantly unaltered feed intake. We have no data to compare the severity of the immune reaction due to the vaccination with that of other studies because we collected no blood samples. Therefore, we cannot conclude why we obtained different results for feed intake compared to other studies. It might be that the amount of pyrogenic substances was not enough to lead to such a strong immune reaction that the appetite would be influenced to a large degree. In addition, the fever period might not have lasted long enough or might have had inappropriate timing to show an effect on the gilts' appetite.

The RA vaccination induced fever for a short period of time and induced mild sickness behavior, such as an increased lying time and a lowered water intake. Thus, we can conclude that the treatment induced a mild defence reaction in the gilts. We were able to establish a quasi-continuous monitoring of the immune reaction by using a commercial temperature logger as a vaginal logger and verified the logger temperature using rectalmeasured temperatures. These measurements enabled us to examine minimal changes in animal behavior and their correlation to the current immune status of the animal. From these results, further investigations might lead to findings regarding early disease symptoms before the disease becomes apparent. The use of a vaccine for foodproducing animals and a commercial logger provide the means for low cost and low risk research in the fields of immunology and health monitoring. There were no obvious side effects observed for either the vaccine or logger. The RA vaccination is especially useful when a mild immune stimulation is sufficient for research questions. However, subsequent studies will be required to investigate which cytokines are discharged and in what amount. 


\section{CONCLUSION}

In conclusion, RA vaccination is suitable as a low risk method for immune stimulation and to induce fever in gilts. Therefore, it was possible to evaluate different behavioral patterns.

\section{ACKNOWLEDGEMENT}

The reaches thank Big Dutchman Pig Equipment $\mathrm{GmbH}$ for their assistance. This project was supported by funds from the German Federal Ministry of Food, Agriculture and Consumer Protection (BMELV) via the Federal Office for Agriculture and Food (BLE) in the framework of the innovation support programme.

\subsection{Ethical Standards}

The authors declare that the experiments comply with the current laws of Germany. The animals were humanely treated both in their day-to-day care by the owner and during our study. Permission from the animal care and use committee of the country was granted.

\section{REFERENCES}

Banji, D., J. Pinnapureddy, O.J. Banji, A. Kumar and K. Reddy, 2011. Evaluation of the concomitant use of methotrexate and curcumin on Freund's complete adjuvant-induced arthritis and hematological indices in rats. Ind. J. Pharmacol., 43: 546-550. DOI: 10.4103/0253-7613.84970

Bland, J.M. and D.G. Altman, 1999. Measuring agreement in method comparison studies. Statist. Methods Med. Res., 8: 135-160. PMID: 10501650

Blood, D.C. and J.A. Henderson, 1983. Veterinary medicine: A Textbook of the Diseases of Cattle, Sheep, Pigs, Goats and Horses. 6nd Edn., Bailliere Tindall, London, ISBN-10: 0702009873, pp: 1310.

Brooks, R.E., R.D. Betz and R.D. Moore, 1978. Injury and repair of the lung: Response to intravenous Freund's adjuvant. J. Pathol., 124: 205-205. PMID: 722384

Burfeind, O., M.V. Keyserlingk, D. Weary, D. Veira and W. Heuwieser, 2010. Short communication: Repeatability of measures of rectal temperature in dairy cows. J. Dairy Sci., 93: 624-627. DOI: 10.3168/jds.2009-2689

Cort, N. and H. Kindahl, 1990. Endotoxin-induced abortion in early pregnant gilts and its prevention by flunixin meglumine. Acta Vet. Scandinavica, 31: 347-358. PMID: 2080779
Dantzer, R., 2001. Cytokine-induced sickness behavior: Where do we stand? Brain Behav. Immunity, 15: 724. DOI: $10.1006 /$ brbi.2000.0613

De Groot, J., G. Kranendonk, M. Fillerup, H. Hopster and W. Boersma et al., 2007. Response to LPS in female offspring from sows treated with cortisol during pregnancy. Physiol. Beha., 90: 612-618. DOI: $10.1016 /$ j.physbeh.2006.11.013

Dumitrescu, A.L., S. Abd El-Aleem, B. Morales-Aza and L.F. Donaldson, 2004. A model of periodontitis in the rat: Effect of lipopolysaccharide on bone resorption, osteoclast activity and local peptidergic innervation. J. Clin. Periodontol., 31: 596-603. DOI: 10.1111/j.1600-051X.2004.00528.x

EC, 2002. Council Regulation (EC) No 1774/2002 of 3 October 2002 laying down health rules concerning animal by-products not intended for human consumption. Official J. Eur. Communities, 273: 1-95.

Fraser, A.F. and J.P. Quine, 1989. Veterinary examination of suffering as a behavior-linked condition. Applied Anim. Behav. Sci., 23: 353-364.

Grouven, U., R. Bender, A. Ziegler and S. Lange, 2007. Vergleich von messmethoden [comparison of measuring methods. Deutsche Medizinische Wochenschrift, 132: e69-e73.

Harden, L.M., I.D. Plessis, J. Roth, L.C. Loram, S. Poole, H.P. Laburn, 2011. Differences in the relative involvement of oeripherally released interleukin (IL6), brain IL-1 beta and prostanoids in mediating lipopolysaccharide-induced fever and sickness behavior. Psychoneuroendocrino. DOI: 10.1016/j.psyneuen. 2010.09.003

Hart, B.L., 1988. Biological basis of the behavior of sick animals. Neurosci. Biobehav. Rev., 12: 123-137.

Hoffmann, G., M. Schmidt, C. Ammon, S. RoseMeierhöfer and O. Burfeind et al., 2013. Monitoring the body temperature of cows and calves using video recordings from an infrared thermography camera. Vet Res. Commun., 37: 91-99. DOI: 10.1007/s11259-012-9549-3

Huynh, T.T.T., A.A. Aarnink, W.J.J. Gerrits, M.J.H. Heetkamp and T.T. Canh et al., 2005. Thermal behavior of growing pigs in response to high temperature and humidity. Applied Anim. Behav. Sci., 91: 1-16. DOI: 10.1016/j.applanim.2004.10.020

Johnson, R.W., 2002. The concept of sickness behavior: A brief chronological account of four key discoveries. Vet. Immunol. Immunopathol., 87: 443450. DOI: 10.1016/S0165-2427(02)00069-7 
Kelley, K.W., R.W. Johnson and R. Dantzer, 1994. Immunology discovers physiology. Veterinary Immunol. Immunopathol., 43: 157-165. DOI: 10.1016/0165-2427(94)90132-5

Kluger, M.J., W. Kozak, C.A. Conn, L.R. Leon and D. Soszynski, 1998. Role of Fever in Disease. In: Molecular Mechanism of Fever, Kluger, M.J., T. Bartfai and C.A. Dinarello (Eds.), Annals of the New York Academy of Sciences, pp: 224-233.

Konsman, J.P., P. Parnet and R. Dantzer, 2002. Cytokine-induced sickness behavior: Mechanisms and implications. Trends Neurosci., 25: 154-159. DOI: 10.1016/S0166-2236(00)02088-9

Leenaars, M. and C.F.M. Hendriksen, 2005. Critical steps in the production of polyclonal and monoclonal antibodies: Evaluation and recommendations. Ilar J., 46: 269-279. PMID: 15953834

Loon, V.J., J. de Grauw, M. van Dierendonck, J. Lami and W. Back et al., 2010. Intra-articular opioid analgesia is effective in reducing pain and inflammation in an equine LPS induced synovitis model. Equine Vet. J., 42: 412-419. DOI: 10.1111/j.2042-3306.2010.00077.x

McCarthy, D.O., M.J. Kluger and A.J. Vander, 1985. Suppression of food intake during infection: Is interleukin-1 involved. Am. J. Clin. Nutrition, 42: 1179-1182. PMID: 3907325

Melchior, D., H. Seve and N.L. Floch, 2004. Chronic lung inflammation affects plasma amino acid concentrations in pigs. J. Anim. Sci., 82: 1091-1099. PMID: 15080331

Melchior, D., N. Meziere, B. Seve and N.L. Floch, 2005. Is tryptophan catabolism increased under indoleamine 2,3 dioxygenase activity during chronic lung inflammation in pigs. Reproduct. Nutr. Dev., 45: 175-183. DOI: 10.1051/rnd:2005013

Nagai, H., H. Arai, F. Ariji, N. Asoo and T. Ishikawa et al., 1957. Morphology and biochemistry of the lung from guinea pig after repeated injections of Freund's complete adjuvant. Lung, 154: 113-123.

Naylor, J.M., R.M. Streeter and P. Torgerson, 2012. Factors affecting rectal temperature measurement using commonly available digital thermometers. Res. Vet. Sci., 92: 121-123. DOI: 10.1016/j.tvsc.2010.10.027

Noh, K.T., K.H. Son, I.D. Jung, H.K. Kang and S.A. Hwang et al., 2012. Protein Kinase C delta (PKC delta)-Extracellular Signal-regulated Kinase $1 / 2$ (ERK1/2) Signaling Cascade Regulates Glycogen Synthase Kinase-3 (GSK-3) Inhibition-mediated Interleukin-10 (IL-10) Expression in Lipopolysaccharide (LPS)-induced Endotoxemia. J. Biol. Chem., 287: 14226-14233. DOI: 10.1074/jbc.M111.308841
Schaefer, A.L., N. Cook, S.V. Tessaro, D. Deregt and G. Desroches et al., 2004. Early detection and prediction of infection using infrared thermography. Canadian J. Anim. Sci., 84: 73-80.

Soede, N.M., W. Hazeleger, J. Broos and B. Kemp, 1997. Vaginal temperature is not related to the time of ovulation in sows. Anim. Reproduct. Sci., 47: 245-252. DOI: 10.1016/S0378-4320(97)00006-7

Stills, H.F., 2005. Adjuvants and antibody production: dispelling the myths associated with Freund's complete and other adjuvants. Ilar J., 46: 280-293. PMID: 15953835

Suthar, V., O. Burfeind, B. Maeder, W. Heuwieser, 2013. Agreement between rectal and vaginal temperature measured with temperature loggers in dairy cows. J. Dairy Res., 80: 240-245. DOI: 10.1017/Soo22029913000071

Swanepoel, T., B.H. Harvey, L.M. Hareden, H.P. Laburn, D. Mitchell, 2011. Dissociation between learning and memory impairment and other sickness behaviors during simulated Mycoplasma infection in rats. Brain Behav. Immun., 25: 1607-1616. DOI: 10.1016/j.bbi.2011.05.008

Timsit, J.F., Y. Dubois, C. Minet, A. Bonadona and M. Lugosi et al., 2011. New challenges in the diagnosis, management and prevention of central venous catheter-related infections. Semin Respir. Crit. Care Med., 32: 139-150. DOI: 10.1055/s-0031-1275526

Tizard, I., 2008. Sickness behavior, its mechanism and significance. Anim. Health Res. Rev., 9: 87-99. DOI: $10.1017 / \mathrm{S} 1466252308001448$

Vickers, L.A., O. Burfeind, M.A.G.V. Keyserlingk, D.M. Veira and D.M. Weary et al., 2010. Technical note: comparison of rectal and vaginal temperatures in lactating dairy cows. J. Dairy Sci., 93: 5246-5251. DOI: $10.3168 /$ jds.2010-3388

Waldron, M.R., A.E. Kulick, A.W. Bell and T.R. Overton, 2006. Acute experimental mastitis is not causal toward the development of energy-related metabolic disorders in early postpartum dairy cows. J. Dairy Sci., 89: 596-610.

Weary, D.M., J.M. Huzzey and M.A.G.V. Keyserlingk, 2009. Board-invited review: Using behavior to predict and identify ill health in animals. J. Anim. Sci., 87: 770-777. DOI: 10.2527/jas.2008-1297

Weary, D.M., L. Niel, F.C. Flower and D. Fraser, 2006. Identifying and preventing pain in animals. Applied Anim. Behav. Sci., 100: 64-76. DOI: 10.1016/j.applanim.2006.04.013 
Wright, K.J., R. Balaji, C.M. Hill, S.S. Dritz and E.L. Knoppel et al., 2000. Integrated adrenal, somatotropic and immune responses of growing pigs to treatment with lipopolysaccharide. J. Anim. Sci., 78: 1892-1899. PMID: 10907832
Zimmermann, W. and H. Plonait, 2004. Erkrankungen des Atmungsapparates Diseases of the Respiratory Tract. In: Lehrbuch der Schweinekrankheiten, Plonait, H., K. Bickhardt and K.H. Waldmann (Eds.), Georg Thieme Verlag, Hannover, Germany, ISBN-10: 3830441045, pp: 142-148. 